\title{
Vacuum Packaging Requirements for MEMS and Characterization Techniques ${ }^{+}$
}

\author{
Luca Mauri *, Anna Della Porta, Alessio Corazza and Marco Moraja \\ SAES Getters S.p.A., viale Italia 77, 20045 Lainate, Italy; anna_della_porta@saes-group.com (A.D.P.); \\ alessio_corazza@saes-group.com (A.C.); marco_moraja@saes-group.com (M.M.) \\ * Correspondence: luca_mauri@saes-group.com; Tel.: +39-0293178417 \\ + Presented at the 4th International Conference nanoFIS 2020-Functional Integrated nano Systems, Graz, \\ Austria, 2-4 November 2020.
}

Published: 15 December 2020

\begin{abstract}
Getter materials are technically proven and industrially well-implemented solutions for maintaining a vacuum inside electronic devices to assure long lifetimes and proper operating conditions. The pressure requirements of some hermetically packaged microelectromechanical systems (MEMS) devices, such as gyroscopes, accelerometers, infrared (IR) bolometers, and digital mirrors, are very stringent. The internal pressure can be as low as in the $10^{-3} \mathrm{mbar}$ range. Due to the desorption phenomena of gaseous species from the internal surfaces, the vacuum inside such hermetically sealed electronic devices tends to degrade over time and, in the worst case, can affect the proper operation of the device. The integration of a special nanostructured getter film is an effective way to preserve and guarantee the performance of such devices. In addition to the getter material, there is also the need to develop and customize analytical techniques for post-process vacuum quality control and reliability checks of hermetic bonding, which are extremely important for the assessment of a device's overall performance, lifetime, and manufacturing process yield.
\end{abstract}

Keywords: MEMS; getter coating; vacuum packaging; hermetic sealing

\section{Introduction}

The packaging of microelectromechanical systems (MEMS) is a key technical challenge, since many devices require a vacuum or well-controlled atmosphere to properly operate and to increase their lifetime. The main challenges in achieving a suitable vacuum level or an adequate gas filling purity are related to the small volume of this type of device and to the high surface-to-volume ratio. The pressure requirements of some hermetically packaged MEMS devices, such as gyroscopes, accelerometers, infrared (IR) bolometers, and digital mirrors, are very stringent in the range of $10^{-3}$ mbar [1-3].

The most used and accepted solution to keep the inner pressure of a hermetically sealed device constant is the getter material [4], which is able to chemically absorb active gases in a vacuum or in an inert gas atmosphere for the entire lifetime of the MEMS devices. Nanostructured getter film that is specifically designed and optimized for such applications can be integrated at the wafer level inside devices. This getter film has the functional property of getting rid of all the gas contaminations coming from the outgassing of internal MEMS surfaces and, to some extent, of managing possible micro-leaks of the sealing, thus ensuring the proper lifetime of the MEMS.

The knowledge of the internal atmospheric composition of microelectromechanical systems (MEMS) is a key issue for deeply investigating the characteristics of these devices [5]. Such information can be obtained using dedicated measurements, which are performed with specific and custom-made experimental apparatuses, which are known as residual gas analysis (RGA) and outgassing tests. 


\section{Getter Technology for MEMS}

The getter film consists of a special Zr-based alloy, whose chemical composition has been optimized to maximize sorption performance and to get a material suitable for becoming active in specific sealing or bonding conditions.

The typical thickness of the patterned getter films at wafer level is in the range of a few microns. The morphology of the getter film has been specifically engineered in order to optimize gas sorption performance. The main gases that can be absorbed by the getter film are $\mathrm{H}_{2}, \mathrm{~N}_{2}, \mathrm{CO}, \mathrm{CO}_{2}, \mathrm{H}_{2} \mathrm{O}, \mathrm{CH}_{4}$, and $\mathrm{O}_{2}$.

The getter film can be activated during the sealing process or the wafer-level bonding process. The different vacuum bonding technologies, such as anodic bonding, eutectic bonding, direct bonding, or glass frit bonding, offer a compatible range of temperatures and bonding times for properly activating the getter material during the wafer-level bonding process.

\section{Characterization Techniques}

The measurement of the vacuum level and the gas composition by means of residual gas analysis (RGA) allows one to obtain useful information about the quality of the bonding process, the hermeticity of the MEMS, and the internal outgassing level; it is possible to measure the leak rate, to extract predictions about device lifetime, and, finally, to investigate the presence of detrimental gas species and their possible removal using a getter film.

The knowledge of the outgassing characteristics of several materials is a fundamental issue in the frame of evacuated sealed devices. With this measurement, in fact, it is possible to obtain different key information related to possible pressure increases inside a device as a function of both the bonding process and the expected lifetime, to perform a proper sizing of the getter material for the specific requirements of the application, and to provide straight indications for the selection of materials and their compatibility with vacuums.

\section{Conclusions}

Creating and maintaining a good vacuum or a high purity of a filling gas in miniaturized systems pose important challenges; special nanostructured getter films have been developed to effectively sorb contaminants and keep proper conditions in hermetically sealed devices. The availability of analytical techniques to determine outgassing rates and to measure residual gas compositions in the systems is a key aspect in supporting the continuous efforts in developing suitable solutions for sealed devices with high reliability and stable performance.

Conflicts of Interest: The authors declare no conflict of interest.

\section{References}

1. Les, C.B. Uncooled IR cameras and detectors: Costing less, scaling up. Photonics Spectra 2010, 44, 22-23.

2. Rogalski, A. Infrared detectors for the future. Acta Phys. Pol. A 2009, 116, 389-406. doi:10.12693/APhysPolA.116.389.

3. Kempe, V. Inertial MEMS: Principles and Practise; Cambridge University Press: Cambridge, UK, 2011. doi:10.1017/CBO9780511933899.

4. Moraja, M.; Amiotti, M. Getters films at wafer level for wafer to wafer bonded MEMS. In Proceedings of the Symposium on Design, Test, Integration and Packaging of MEMS/MOEMS 2003, Cannes, France, 7 May 2003.

5. Costello, S.; Desmulliez, M.P.Y. Hermeticity Testing of MEMS and Microelectronic Packages; Artech House: Boston, MA, USA, 2013.

Publisher's Note: MDPI stays neutral with regard to jurisdictional claims in published maps and institutional affiliations. 